Tsuchiya et al., Page 1

\title{
A Multi-center Phase II Study of Adjuvant Chemotherapy with Oral Fluoropyrimidine S-1 for Non-Small Cell Lung Cancer: High Completion and Survival Rates
}

Tomoshi Tsuchiya ${ }^{1}$, Takeshi Nagayasu ${ }^{1}$, Naoya Yamasaki ${ }^{1}$, Keitaro Matsumoto ${ }^{1}$, Takuro $5 \quad$ Miyazaki $^{1}$, Tsutomu Tagawa ${ }^{2}$, Akihiro Nakamura $^{3}$, Hiroyuki Minami ${ }^{3}$, Hideki Taniguchi ${ }^{4}$, Shinji Akamine ${ }^{5}$, Hiroshi Hisano ${ }^{6}$, and Yoshitaka Taniguchi ${ }^{7}$

${ }^{1}$ Division of Surgical Oncology, Department of Translational Medical Sciences, Nagasaki University Graduate School of Biomedical Sciences, 1-7-1 Sakamoto, Nagasaki City 852-8501, Japan

${ }^{2}$ Department of Surgery, National Hospital Organization Nagasaki Medical Center, Omura City, Japan

${ }^{3}$ Department of Surgery, Sasebo Municipal Hospital, Sasebo City, Japan

${ }^{4}$ Department of Surgery, The Japanese Red Cross Nagasaki Genbaku Hospital, Nagasaki City, 15 Japan

${ }^{5}$ Department of Chest Surgery, Oita Prefectural Hospital, Oita City, Japan

${ }^{6}$ Department of Surgery, Saiseikai Nagasaki Hospital, Nagasaki City, Japan

${ }^{7}$ Fukuda Geka Hospital, Sasebo City, Japan

Funding sources : none

Running title: Adjuvant S-1 chemotherapy for lung cancer

Corresponding author: Takeshi Nagayasu, $\mathrm{MD}, \mathrm{PhD}$

25 Division of Surgical Oncology, Department of Translational Medical Sciences 
Tsuchiya et al., Page 2

Nagasaki University Graduate School of Biomedical Sciences

1-7-1 Sakamoto, Nagasaki City 852-8501, Japan

Phone: +81-95-849-7304; Fax: +81-95-849-7306

E-mail: nagayasu@nagasaki-u.ac.jp

Number of words: abstract, 224; manuscript, 2892

Unique trial ID number: NCT01459185 or R000007795 (UMIN ID)

Trial registration date: October 21, 2011 
Tsuchiya et al., Page 3

\section{Conflict of Interest}

All authors have no conflicts of interest. 
Tsuchiya et al., Page 4

\section{MicroAbstract}

40 As oral chemotherapy might reduce physiological and psychological burdens on patients, we conducted a feasibility study using S-1, an oral fluoropyrimidine, as postoperative chemotherapy in 50 patients with curatively resected stage IB-IIIA non-small cell lung cancer. The completion rate was $72.0 \%$ and the 3 -year relapse-free survival rate was $69.4 \%$. This protocol seems feasible and may be sufficient to prevent recurrence. 
Tsuchiya et al., Page 5

\section{Abstract}

Background: Oral adjuvant chemotherapy without hospitalisation might reduce the physiological and psychological burden on patients if effectiveness could be guaranteed. We conducted a multi-center feasibility study using S-1, an oral derivative of 5-fluorouracil, as postoperative adjuvant chemotherapy in patients with curatively resected pathologically stage IB-IIIA non-small cell lung cancer. Patients and Methods: Adjuvant chemotherapy comprised eight courses (4-week administration, 2-week withdrawal) of S-1 at 80-120 mg/body/day. Fifty-one patients from seven institutions were enrolled in this pilot study, from June 2005 to March 2007. The primary endpoint was the completion rate of scheduled adjuvant chemotherapy. Secondary endpoints were the incidence and grade of adverse reactions. Results: Fifty patients were eligible. The completion rate for the planned eight courses of S-1 administration was 72.0\% (36 patients). Total percentage administration amount was 71.1\%. Grade 3 adverse reactions such as neutropenia (4.0\%), anorexia (4.0\%), thrombopenia (2.0\%), anemia (2.0\%), elevated total bilirubin (2.0\%), hypokalemia (2.0\%), nausea (2.0\%) and diarrhoea (2.0\%) were observed, but no grade 4 adverse effects were encountered. Overall and relapse-free survival rates at 3 years were $87.7 \%$ and $69.4 \%$, respectively. Conclusion: Postoperative 1-year administration of S-1 seems feasible as oral adjuvant chemotherapy for lung cancer. The oral formulation and low incidence of adverse reactions permit treatment on an outpatient basis. The present study would be reasonable to follow up with a properly powered phase III trial.

Keywords: Non-small cell lung cancer, Adjuvant chemotherapy, Fluoropyrimidine, S-1, Feasibility study 
Tsuchiya et al., Page 6

\section{Introduction}

The results of surgical treatment for lung cancer have been improved by early detection and meticulous surgical procedures. However, we still face recurrence in patients with advanced lung cancer, even after extended surgical treatment. Since 2004, clinical research studies have established the efficacy of adjuvant chemotherapy in post-operative patients with stage IB-IIIA non-small cell lung cancer (NSCLC). ${ }^{1-3}$ Standard regimens for adjuvant chemotherapy currently use intravenous administration of a platinum doublet. However, oral adjuvant chemotherapy with uracil-tegafur, which improved survival among patients with completely resected stage IB adenocarcinoma, allows completion of the regimen with only mild adverse reactions. ${ }^{1}$ Such oral drugs enable patients to undergo treatment on an outpatient basis, and are suitable for maintaining patient quality of life.

S-1 is a novel oral fluoropyrimidine derivative consisting of tegafur (FT) and two modulators, 5-chloro-2,4-dihydroxypyridine (CDHP) and potassium oxonate (Oxo), in a molar ratio of 1:0.4:1. ${ }^{4} \mathrm{FT}$ is a prodrug of 5-fluorouracil (5-FU) and CDHP is a reversible competitive inhibitor of dihydropyrimidine dehydrogenase (DPD; EC1.3.1.2), an enzyme involved in the degradation of 5-FU. Degradation of FT-derived 5-FU is thus efficiently inhibited by CDHP, and 5-FU remains in plasma and tumor tissue longer and at higher levels than when low-dose 5-FU is continuously infused intravenously. The major toxicities associated with fluoropyrimidines are diarrhoea and mucositis. ${ }^{5}$ Oxo is a reversible competitive inhibitor of orotate phosphoribosyltransferase (EC2.4.2.10), a phosphoenzyme for 5-FU, and is distributed at high levels in the gastrointestinal (GI) tract after oral administration, reducing GI toxicity caused by 5 -FU. ${ }^{6}$

Response rates for platinum doublet therapy in patients with advanced lung cancer are 30-33\%. ${ }^{7}$ Conversely, S-1 showed a 22\% response rate for advanced NSCLC in a previous phase II trial, ${ }^{8}$ raising the question of whether S-1 has sufficient power as adjuvant 
chemotherapy. In that regard, the therapeutic strategy with oral fluoropyrimidine differs from that with intensive platinum doublets. Despite showing a response rate of only $7 \%$ for advanced NSCLC, uracil-tegafur, another oral fluoropyrimidine, could be administered long-term (2 years) and, thus, could allow administration of sufficient amounts to prevent recurrence or metastasis, because the drug has extremely low toxicity and is easily continued as an oral medication. ${ }^{9}$ Although the exact mechanisms of action accounting for the efficacy of uracil-tegafur treatment in the postoperative adjuvant setting remain unclear, long-term uracil-tegafur administration may inhibit the development of postoperative recurrence through antiangiogenic effects in addition to direct cytotoxic effects. ${ }^{10}$ In terms of S-1-based adjuvant chemotherapy, in the field of gastric cancer, a feasibility study has already been performed and achieved a high completion rate of $60 \%$ and a favorable drug compliance rate of $70 \% .{ }^{11}$ Furthermore, a phase III randomized study comparing surgery alone to surgery plus adjuvant chemotherapy using S-1 was ongoing in $2005 .{ }^{12}$ Given this background, we presumed that if long-term administration could be achieved in the postoperative adjuvant setting, similar factors would be applicable even for stage II to IIIA disease because of the higher response rate compared to uracil-tegafur.

Based on similar notions, a feasibility study for adjuvant chemotherapy was reported by Yano et al. in $2010 .^{13}$ In that study, $56.7 \%$ of patients finished the regimen. Postoperative administration of S-1 for 6 months was thus considered feasible as adjuvant chemotherapy for NSCLC. However, the administration period of 6 months is half the reported duration of the adjuvant chemotherapy with $\mathrm{S}-1^{12}$ and the completion rate is unsatisfactorily low despite the mildness of adverse reactions. Moreover, survival data from the study have yet to be reported. 
Tsuchiya et al., Page 8

To confirm the feasibility of 1-year administration of S-1 and analyse the effect of the intervention on prognoses, a multi-center phase II clinical trial was conducted in seven facilities.

\section{Patients and Methods}

\section{Patient Eligibility}

Patient eligibility required compliance with the following criteria: NSCLC with histological proof; pathological stage IB, II, or IIIA NSCLC (according to the fifth edition of UICC/AJCC 1997) ${ }^{14}$ after complete resection; no prior treatment except for surgery; age >20 and $<80$ years, with sufficient oral intake; and performance status (PS) 0 or 1 . Patients also had to have adequate organ function $\left(3500 \leq\right.$ leukocytes $\leq 12,000 / \mathrm{mm}^{3}$; thrombocytes, $\geq 100,000 / \mathrm{mm}^{3}$; total bilirubin, $\leq 1.5 \mathrm{mg} / \mathrm{dl}$; aspartate aminotransferase and alanine aminotransferase, less than twice the normal limits at each institution; blood urea nitrogen, $\leq 25 \mathrm{mg} / \mathrm{dl}$; creatinine, less than the normal limits at each institution; and creatinine clearance (Ccr) as estimated by the Cockcroft-Gault formula, $\geq 50 \mathrm{ml} / \mathrm{min}$ ). Patients with a history of drug hypersensitivity, serious surgical or non-surgical complications, or active secondary cancer were excluded. Pregnant or lactating women were likewise excluded.

\section{Treatment Schedule}

Chemotherapy comprised eight courses (4-week administration, 2-week withdrawal) of S-1 (FT, gineracil, oteracil potassium; Taiho Pharmaceutical, Tokyo, Japan) at 80-120 $\mathrm{mg} /$ body/day according to body surface area (BSA): BSA $<1.25 \mathrm{~m}^{2}, 80 \mathrm{mg} / \mathrm{day} ; \mathrm{BSA} \geq 1.25$ $\mathrm{m}^{2}$ but $<1.5 \mathrm{~m}^{2}, 100 \mathrm{mg} /$ day; and BSA $\geq 1.5 \mathrm{~m}^{2}, 120 \mathrm{mg} /$ day. S-1 was administered orally, twice daily after meals, starting within 4 weeks after surgery. Every 6 weeks, patients visited the hospital and drug compliance was checked from the treatment diary. Subjective 
Tsuchiya et al., Page 9

symptoms, clinical experiment including hematological toxicities and tumor markers were also confirmed. The administration dose for the next course was determined after checking these data. Doses were modified in accordance with the following guidelines. When adverse

\section{Study Design and Statistical Analysis}

This trial was non-blinded and open-label. The primary endpoint was the completion rate of the scheduled adjuvant chemotherapy. Secondary endpoints were the incidence and grade of adverse reactions. The number of patients to be enrolled in this study was calculated as 55 . Assuming a completion rate of $70 \%$, with a planned eligible sample size of 50 patients, the 95\% confidence interval (CI) for the completion rate was estimated to range from $55 \%$ to 
Tsuchiya et al., Page 10

$82 \%$. This completion rate of $70 \%$ means that $70 \%$ of patients would complete 48 weeks of planned chemotherapy.

The Kaplan-Meier method was used to estimate the time-to-event functions of relapse-free survival and overall survival. Relapse-free survival has been defined as the time from the date of the start of treatment to the date of disease progression or death (whichever occurs first) or the date of last contact. Overall survival has been defined as the time from the date of the start of treatment to the date of death or last contact. The log-rank test was used to test for possible differences between estimated time-to-event curves.

\section{Ethics}

This study was approved by the institutional review board at each site. Patients selected whether they would participate in the trial or not after detailed explanation and written informed consent was obtained from all patients prior to enrolment. In terms of one institution (Nagasaki University Hospital), 52 patients were referred to the trial. Among the referred patients, 15 patients participated in the trial, 14 patients preferred to received uracil-tegafur (p-stage IB), and 23 patients preferred to received standard chemotherapy.

\section{Results}

\section{Patient Characteristics}

A total of 51 patients were initially enrolled in the present study. One patients were ineligible, who rescinded consent to enter the trial before administration of S-1. Table 1 shows the characteristics of the 50 eligible patients. The median age of patients was 71.0 years (range, 32-80 years). Lobectomy was performed in all patients.

\section{Drug Compliance}


Tsuchiya et al., Page 11

Table 2 shows drug compliance in each course and reasons for discontinuation of drug administration. The planned eight courses of S-1 were administered to 36 patients $(72.0 \%)$. Among these 36 patients, 25 patients received dose reduction (69.4\% of 36 patients). Thirteen patients discontinued drug administration because of anorexia, diarrhoea, thrombocytopenia, elevated total bilirubin, fever, or stomatitis $(n=5)$. Non-iatrogenic reasons for discontinuation included patient refusal ( $n=6)$, transfer to a different hospital $(n=1)$ and administrative errors $(n=1)$. The discontinued case occurred within the third course and drug compliance was maintained at $>85 \%$ (85.4-99.4\%) in every course. In the total group of 50 patients, the percentage of actual days on which S-1 was administered against the total number of planned administration days (28 days $\times 8$, i.e., 224 days) was $77.3 \%$. Concerning the amount of drug administered, the compliance rate was $71.1 \%$.

\section{Adverse Reactions and Dose Reduction}

Table 3 shows a summary of the adverse reactions encountered. Among the laboratory findings-based adverse reactions, increased serum total bilirubin was the most frequent, occurring in 8 of the 50 patients (16.0\%), followed by thorombocytopenia (12.0\%), anemia (12.0\%), and leukocytopenia (10.0\%). Among the clinical findings-based adverse reactions, anorexia was the most frequent (42.0\%), followed by nausea (12.0\%), diarrhoea (6.0\%), pigmentation changes (6.0\%), stomatitis (4.0\%), malaise (4.0\%), and constipation (4.0\%). Concerning the incidence and grade of laboratory findings-based adverse reactions, grade 3 adverse reactions were seen with neutropenia, thorombocytopenia, anemia, increased serum total bilirubin, and hypokalemia. No grade 4 adverse reactions were identified. In clinical findings-based adverse reactions, grade 3 adverse reactions were observed with anorexia, nausea, and diarrhoea. Again, no grade 4 adverse reactions were encountered. 
Tsuchiya et al., Page 12

The completion rate was $86.7 \%$ among cases without adverse reactions (Table 4). When adverse reactions occurred, completion rate decreased to $65.7 \%$. However, dose reduction clearly increased the completion rate (79.2\%). When administration was restarted without a dose reduction, the completion rate was significantly lower (36.4\%).

\section{Survival}

Among the 50 patients followed for survival information, only 13 had died and 37 were still alive at the time of analysis. Median follow-up time was 49.0 months (range, 7.3-66.4 months). At the time of analysis, overall survival rate at 36 months was $87.7 \%$ (95\%CI, 75.2-94.4) (Figure 1). Of the 13 patients who died, 8 had experienced a documented relapse before death. Four patients died of brain infarction, pneumonia, newly developed malignant lymphoma, or interstitial pneumonia 9 months after the discontinuation of S-1 administration. A total of 14 patients relapsed, and the relapse-free survival rate at 36 months was $69.4 \%$ (95\%CI, 55.2-80.6) (Figure 1). Among the patients who experienced relapse, 6 patients experienced intrathoracic relapse, including five with regional lymphatic metastasis and one with dissemination, and 8 patients showed distant relapse alone.

\section{Discussion}

The present study was undertaken to confirm the feasibility of 1-year oral adjuvant chemotherapy with S-1 after standard resection for NSCLC. The completion rate for the planned eight courses of S-1 administration was $72.0 \%$, which compares favourably to the chemotherapy compliance seen on trials of cisplatin-based adjuvant therapies that have ranged from $45 \%$ to $76 \%$ of the intended dose. ${ }^{11-13}$ Toxicity in the present study was significantly less compared with the other regimen. No grade 4 adverse reactions were observed throughout the eight courses. Only six grade 3 hematological and four grade 3 
non-hematological adverse events were encountered (20.0\% of total). The most common adverse reaction was grade 1 anorexia, in $42.0 \%$ of patients, and administration for outpatients was easily continued. Compared to postoperative adjuvant chemotherapy study using uracil-tegafur, another oral fluoropyrimidine, the frequency of grade 3 adverse reactions was less than $4 \%$. The most common adverse reaction was grade 1 gastrointestinal toxicity, including anorexia, nausea and vomiting in around $10 \%$ of patients, representing an extremely low frequency. ${ }^{1}$ Conversely, studies of platinum-based postoperative adjuvant chemotherapy have indicated that the frequency of grade 3 or more adverse reactions was more than $69 \%$ even with carboplatin-based therapies. ${ }^{16,18}$ Accordingly, S-1 is considered to cause intermediate adverse reactions, allowing acceptable compliance. Furthermore, the possibility of outpatient treatment with S-1 is convenient for both doctors and patients.

In the present study, the total percentage administration days and percentage administration dose were $77.3 \%$ and $71.1 \%$. Whether a dose reduction of $70 \%$ allows sufficient power to prevent recurrence of lung cancer remains unclear. In analysis of a phase III study of postoperative gastric cancer, ${ }^{12}$ when protocol completion cases were divided into two groups according to compliance with S-1 administration, the 5-year survival curves for patients with $\geq 90 \%$ compliance and patients with $70 \%$ to $<90 \%$ compliance overlapped (in-house experimental data; Taiho Pharmaceutical). We therefore believe a dose reduction of 70\% provides sufficient adjuvant chemotherapy for lung cancer with S-1, as in gastric cancer. Further studies and long-term observations are necessary to clarify the remaining issues.

The regimen in the present study was based on the seminal phase III randomized study in postoperative gastric cancer. ${ }^{12}$ Among the 517 patients in the safety population who received S-1, treatment was continued for 12 months in 340 patients (65.8\%). In the present study, completion rates were $8 \%$ or more higher than those from the study in gastric cancer. In addition, our results showed incidences of hematological and non-hematological adverse 
reactions were both lower than in the gastric cancer study. As patients in the gastric cancer study displayed rather advanced disease and received D2 or more aggressive gastrectomy with frequent combined organ resections, patients who undergo standard resection for lung cancer might show better general and intestinal conditions for oral chemotherapy.

In a recent feasibility study of adjuvant chemotherapy with S-1 for NSCLC, ${ }^{13}$ the administration period of 6 months and the cycle of 2-week administration and 1-week withdrawal differed from the protocol applied in our study. That study demonstrated no hematological or non-hematological grade 4 adverse reactions throughout the eight courses, very similar to our study. Conversely, completion rate of the planned eight courses of S-1 administration was $56.7 \%$. The reason for the relatively low completion rate was attributed to the high age of patients and the high incidence of patients declining to continue treatment. ${ }^{13}$ In the present study, dose reductions were performed without hesitation. When adverse reactions were encountered, dose reduction obviously improved the completion rate to $79.2 \%$, compared to $36.4 \%$ without dose reduction. As a result, we achieved a high completion rate of $72.0 \%$. The duration of S-1 administration is another area of discrepancy. Administration of 5-FU is known to be more effective in producing direct cytotoxic effects against human tumor cells using lower doses for longer time periods than using higher doses for shorter times. ${ }^{19}$ Our opinion is that at least a year of S-1 is warranted, unless clinical evidence to the contrary is identified.

The overall survival rate among patients with stage IB resected NSCLC was similar to that among patients with stage IIA or more resected NSCLC (data not shown). These data indicate that oral S-1 treatment might have sufficient power to improve survival even in postoperative patients with severe stage NSCLC. Further follow-up survival data are needed for the present study. In addition, randomized phase II and III clinical trials of adjuvant chemotherapy containing S-1 for NSCLCs (WJOG4107 and JCOG0707) are ongoing. The 
JCOG0707 phase III study is comparing survival data and compliance between uracil-tegafur and S-1 for stage IA ( $>2 \mathrm{~cm}$ ) and IB postoperative patients. The results will provide more reliable data on whether S-1 alone is worthwhile as an option for adjuvant chemotherapy.

One limitation of the present study was the difficulty in confirming true drug compliance. We checked drug compliance from the treatment diary every 6 weeks when the patient visited the hospital, but had no way of ensuring that the patient had made true declarations regarding drug intake. Although most seminal studies have not mentioned this point and one study applied a similar method, ${ }^{1}$ investigators must keep in mind that all such oral administration studies conducted on an outpatient basis carry this problem in confirming true drug compliance.

Although S-1 is not well known in Western countries, various clinical trials of S-1-based chemotherapy have been performed or are ongoing for advanced NSCLC, particularly in Japan. ${ }^{20}$ Among chemotherapy-naïve patients with advanced NSCLC, a phase III trial by the West Japan Oncology Group showed oral S-1 plus carboplatin was non-inferior in terms of overall survival when compared to paclitaxel plus carboplatin. ${ }^{21}$ Comparisons of 5-FU-related enzymes of NSCLC in such patients have indicated that low expression of thymidylate synthase (TS) and dihydropyrimidine dehydrogenase (DPD) are associated with better survival in S-1 plus CBDCA therapy, but not in PTX and CBDCA therapy. ${ }^{22}$ As S-1 is a prodrug of 5-FU, we believe the expression of TS, a 5-FU-targeting enzyme, and DPD, a 5-FU-degrading enzyme, are important in determining susceptibility to S-1. Further study of rational differences in 5-FU-related enzymes might be necessary, as expressions of TS and DPD might differ in NSCLC between Western and Eastern populations.

\section{Conclusion}


Tsuchiya et al., Page 16

Postoperative 1-year oral administration of S-1 seems feasible as an adjuvant chemotherapy for lung cancer. A high completion rate was achieved when administration doses were decreased by one rank when adverse events were encountered. The oral formulation and low incidence of adverse reactions permit treatment on an outpatient basis. The present findings suggest that follow-up with a properly powered phase III study comparing treatment using S-1 to the standard of care for adjuvant chemotherapy would be reasonable. 
Tsuchiya et al., Page 17

\section{Clinical Practice Points}

The current standard regimen for adjuvant chemotherapy of NSCLC is intravenous administration of a platinum doublet. However, a seminal study indicated adjuvant chemotherapy with uracil-tegafur, an oral fluoropyrimidine, could improve survival among patients with completely resected stage IB adenocarcinoma. The biggest advantage of such therapy is the low toxicity and easy continuation as oral medication, which can allow long-term administration in amounts sufficient to prevent recurrence. The anti-tumor mechanisms of oral fluoropyrimidine are presumed to differ from those of platinum doublets; long-term administration can inhibit the development of postoperative recurrence through antiangiogenic effects as well as by direct cytotoxic effects.

S-1 is a novel oral fluoropyrimidine derivative consisting of the 5-fluorouracil prodrug tegafur (FT) and two modulators. A modulator of 5-chloro-2,4-dihydroxypyridine (CDHP) is a reversible competitive inhibitor of dihydropyrimidine dehydrogenase (DPD), an enzyme involved in the degradation of 5-FU. As S-1 shows 180-times stronger DPD-inhibiting effect and a higher response rate from patients than uracil-tegafur (22\% vs. 7\% for advanced NSCLC), we considered this therapy would likely prove beneficial for patients with surgically resected pathological IB to IIIA NSCLC.

The new findings of the present study were that we could achieve a favorable completion rate for 1-year S-1-based adjuvant chemotherapy and also showed the possibility of good prognosis for stage IB to IIIA NSCLC in an adjuvant setting. The clinical impact in the foreseeable future is that the present study confirmed S-1-based adjuvant chemotherapy as worthy of follow-up in a properly powered phase III study comparing with the standard of care for adjuvant chemotherapy. 
Tsuchiya et al., Page 18

\section{Acknowledgements}

We wish to thank Dr. Sumihisa Honda for providing statistical advice. We are also grateful to Taiho Pharmaceutical for technical support and invaluable assistance.

\section{Disclosures}

All authors report that they have no relevant relationships to disclose. 
Tsuchiya et al., Page 19

\section{References}

1. Kato H, Ichinose $\mathrm{Y}$, Ohta $\mathrm{M}$, et al. A randomized trial of adjuvant chemotherapy with uracil-tegafur for adenocarcinoma of the lung: Japan Lung Cancer Research Group on Postsurgical Adjuvant Chemotherapy. N Engl J Med 2004; 350:1713-21

2. Arriagada R, Bergman B, Dunant A, et al. Cisplatin-based adjuvant chemotherapy in patients with completely resected non-small-cell lung cancer: International Adjuvant Lung Cancer Trial Collaborative Group. N Engl J Med 2004; 350:351-60

3. Winton T, Livingston R, Johnson D, et al. Vinorelbine plus cisplatin vs. observation in resected non-small-cell lung cancer: National Cancer Institute of Canada Clinical Trials Group; National Cancer Institute of the United States Intergroup JBR.10 Trial Investigators. $N$ Engl J Med 2005; 352:2589-97

4. Shirasaka T, Shimamato Y, Ohshimo H, et al. Development of a novel form of an oral 5-fluorouracil derivative (S-1) directed to the potentiation of the tumor selective cytotoxicity of 5-fluorouracil by two biochemical modulators. Anti-Cancer Drugs 1996; $7: 548-57$

5. Vogelzang NJ. Continuous infusion chemotherapy: a critical review. J Clin Oncol 1984; 2:1289-304

6. Shirasaka T, Shimamoto Y, Fukushima M. Inhibition by oxonic acid of gastrointestinal toxicity of 5-fluorouracil without loss of its antitumor activity in rats. Cancer Res 1993; $53: 4004-9$

7. Kubota K. The Four-Arm Cooperative Study (FACS) for advanced non-small-cell lung cancer (NSCLC). Proc Am Soc Clin Oncol 2004; 22:254

8. Kawahara M, Furuse K, Segawa Y, et al. Phase II study of S-1, a novel oral fluorouracil, in advanced non-small-cell lung cancer: S-1 Cooperative Study Group (Lung Cancer Working Group). Br J Cancer 2001; 85(7):939-43 
Tsuchiya et al., Page 20

9. Ota K, Taguchi T, Kimura K. Report on nationwide pooled data and cohort investigation in UFT phase II study. Cancer Chemother Pharmacol 1988; 22:333-8.

10. Basaki Y, Chikahisa L, Aoyagi K, et al. Gamma-hydroxybutyric acid and 5-fluorouracil, metabolites of UFT, inhibit the angiogenesis induced by vascular endothelial growth factor. Angiogenesis 2001; 4:163-73

11. Kinoshita T, Nashimoto A, Yamamura Y. Feasibility study of adjuvant chemotherapy with S-1 (TS-1;tegafur,gimeracil,oteracil potassium) for gastric cancer. Gastric Cancer 2004; 7:104-9

12. Sakuramoto S, Sasako M, Yamaguchi T, et al. Adjuvant Chemotherapy for Gastric Cancer with S-1, an Oral Fluoropyrimidine: ACTS-GC Group. N Engl J Med 2007; 357(18):1810-20

13. Yano T, Yamazaki K, Maruyama R, et al. Feasibility study of postoperative adjuvant chemotherapy with S-1 (tegaful, gimeracil, oteracil potassium) for non-small cell lung cancer-LOGIK 0601 study: Lung Oncology Group in Kyushu (LOGIK). Lung Cancer $2010 ; 67: 184-7$

14. Mountain CF. Revisions in the International System for Staging Lung Cancer. Chest $1997 ; 111: 1707-10$

15. Dunant A, Pignon JP, Le Chevalier T. Adjuvant chemotherapy for non-small cell lung cancer: contribution of the International Adjuvant Lung Trial. Clin Cancer Res 2005; 11:5017s-21s

16. Douillard JY, Rosell R, De Lena M, et al. Adjuvant vinorelbine plus cisplatin versus observation in patients with completely resected stage IB-IIIA non-small cell lung cancer (Adjuvant Navelbine International Trialist Association [ANITA]): a randomized controlled trial. Lancet Oncol 2006; 7: 719-27 
Tsuchiya et al., Page 21

22. Takeda M, Okamoto I, Hirabayashi N, et al. Thymidylate synthase and dihydropyrimidine dehydrogenase expression levels are associated with response to S-1 plus carboplatin in advanced non-small cell lung cancer. Lung Cancer 2011; 73:103-9 
Tsuchiya et al., Page 22

$425 \quad$ Figure Legends

Figure 13 -year overall survival and relapse-free survival rates 
Figure 1

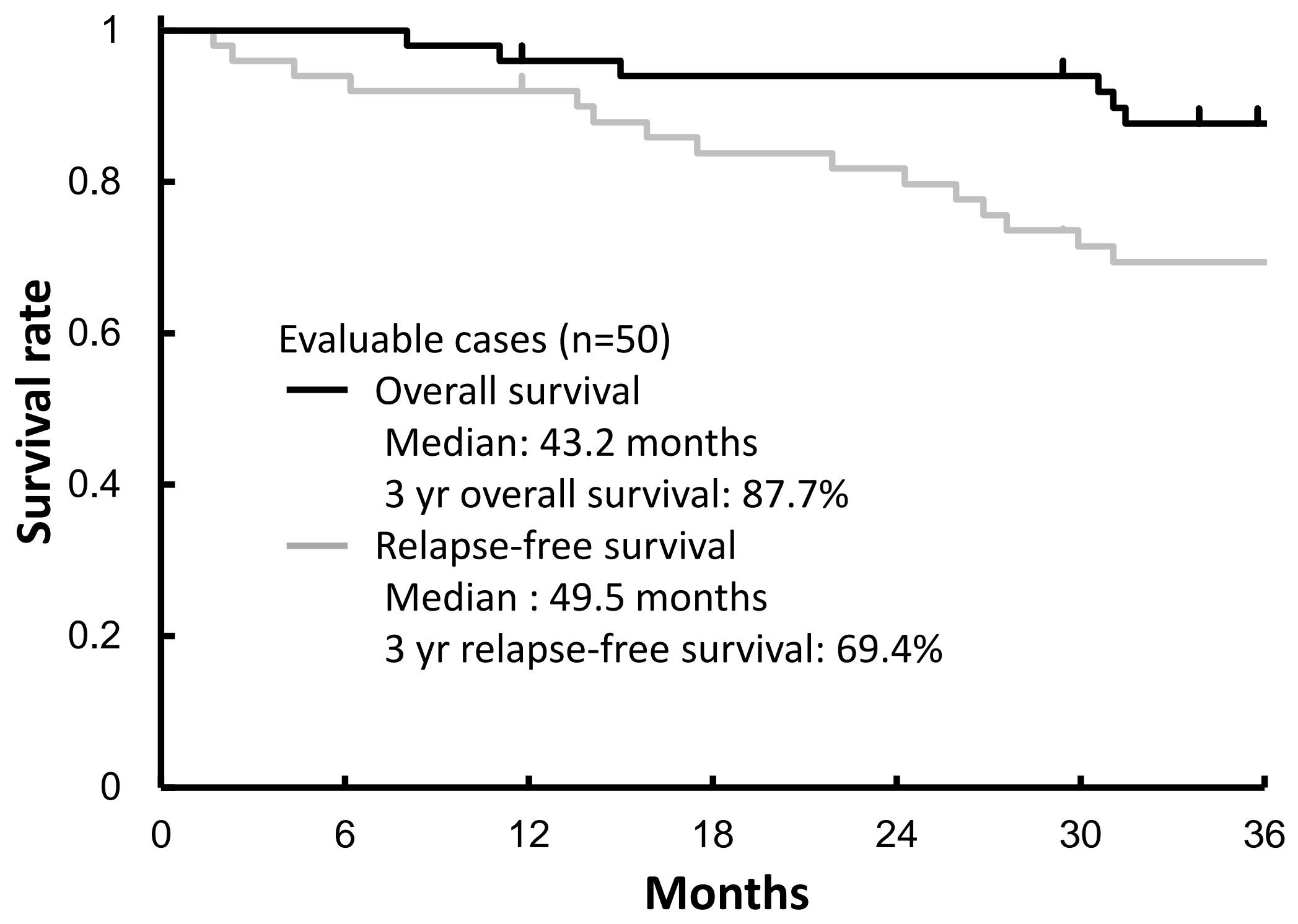


Table 1 Patient Characteristics $(\mathrm{n}=50)$

\begin{tabular}{|c|c|c|c|}
\hline Variables & & $\mathrm{n}$ & Percentage \\
\hline \multirow[t]{2}{*}{ Sex } & Male & 34 & 68.0 \\
\hline & Female & 16 & 32.0 \\
\hline \multirow[t]{5}{*}{ Age (years) } & $<50$ & 4 & 8.0 \\
\hline & $50-59$ & 8 & 16.0 \\
\hline & $60-69$ & 11 & 22.0 \\
\hline & $\geq 70$ & 27 & 54.0 \\
\hline & \multicolumn{3}{|l|}{ Mean, 66.6; median 71.0} \\
\hline Type of resection & Lobectomy & 50 & 100.0 \\
\hline \multirow[t]{2}{*}{$\begin{array}{l}\text { Lymph node } \\
\text { dissection }\end{array}$} & ND1 & 2 & 4.0 \\
\hline & ND2 & 48 & 96.0 \\
\hline \multirow[t]{3}{*}{ Histology } & Adenocarcinoma & 29 & 58.0 \\
\hline & SCC & 19 & 38.0 \\
\hline & Others & 2 & 4.0 \\
\hline \multirow[t]{4}{*}{$\begin{array}{l}\text { Pathological TNM } \\
\text { stage }\end{array}$} & IB & 28 & 56.0 \\
\hline & IIA & 10 & 20.0 \\
\hline & IIB & 5 & 10.0 \\
\hline & IIIA & 7 & 14.0 \\
\hline
\end{tabular}

SCC, squamous cell carcinoma 
Table 2 Drug Compliance (each course) $(\mathrm{n}=50)$

\begin{tabular}{|c|c|c|c|}
\hline Course & $\begin{array}{l}\text { No. of patients } \\
\text { entering course }\end{array}$ & $\%$ & Reason for discontinuation \\
\hline \multirow[t]{3}{*}{1} & 50 & 100 & \\
\hline & & & Grade 2 stomatitis (patient refusal) \\
\hline & & & Recurrence \\
\hline \multirow[t]{10}{*}{2} & 48 & 96.0 & \\
\hline & & & Grade 3 thrombocytopenia \\
\hline & & & Grade2 thrombocytopenia and \\
\hline & & & Grade 1 fever \\
\hline & & & Grade 2 anorexia (patient refusal) \\
\hline & & & Grade 2 anorexia (patient refusal) \\
\hline & & & Grade 3 anorexia \\
\hline & & & Grade 3 diarrhoea \\
\hline & & & Grade 3 elevated total bilirubin \\
\hline & & & Grade 2 anorexia (patient refusal) \\
\hline \multirow[t]{5}{*}{3} & 40 & 80.0 & \\
\hline & & & Grade 1 anorexia and Grade 1 elevated \\
\hline & & & total bilirubin (patient refusal) \\
\hline & & & Patient refusal \\
\hline & & & Change of hospital \\
\hline 4 & 37 & 74.0 & \\
\hline 7 & & & Stopped administration by mistake \\
\hline 8 & 36 & 72.0 & \\
\hline Complete & & & \\
\hline
\end{tabular}


Table 3 Adverse Reactions $(n=50)$

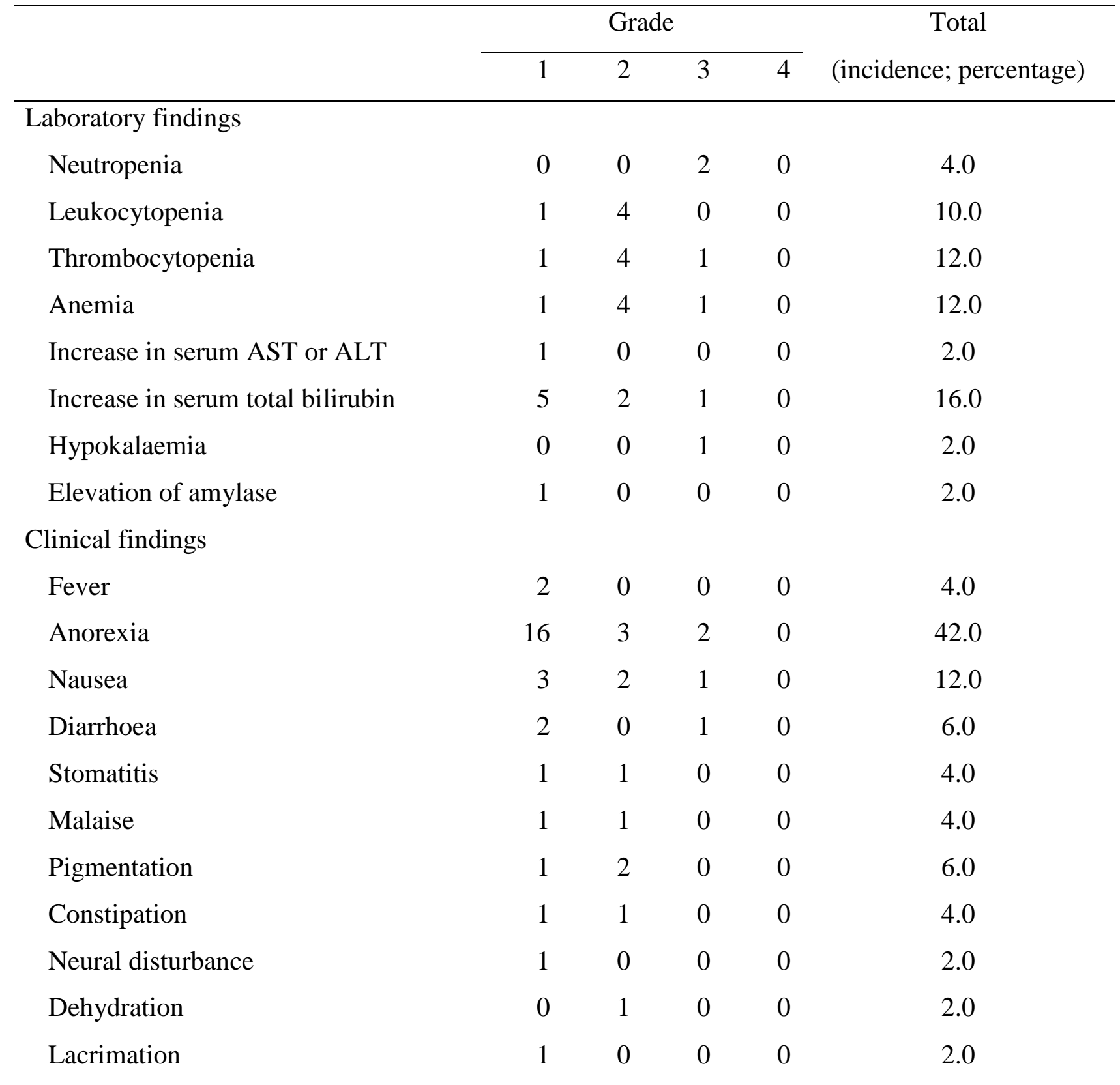

Grade 2 or more thrombocytopenia and other adverse reactions of Grade 3 or more match the criteria for dose reduction 
Table 4 Effect of Dose Reduction on Adverse Reactions $(n=50)$

\begin{tabular}{cccc}
\hline & $\mathrm{n}$ & Completed cases & Completion rate (\%) \\
\hline Without adverse reaction & 15 & 13 & 86.7 \\
With adverse reactions & 35 & 23 & 65.7 \\
with dose reduction & 24 & 19 & 79.2 \\
without dose reduction & 11 & 4 & 36.4 \\
\hline
\end{tabular}

